\title{
Diferencias Morales y Fronteras Sociales. Los Límites de la Inclusión en la Educación Sexual de los Colegios Católicos de Santiago
}

\section{Moral Differences and Social Borders. The Limits of Inclusion in Catholic School's Sexual Education in Santiago}

Pablo Astudillo *

Universidad Alberto Hurtado, Chile

\begin{abstract}
En los colegios católicos de élite, la educación sexual intenta regular la intimidad y el carácter personal, para así dar forma a un individuo capaz de orientarse a sí mismo y su sexualidad hacia un fin determinado. En el presente artículo examinaremos el resultado de una investigación cualitativa desarrollada en 16 establecimientos católicos de la ciudad de Santiago. A partir de 53 entrevistas a orientadores y psicólogos a cargo de la educación sexual, dos grupos focales que convocaron a 23 adolescentes de dichos colegios y observaciones etnográficas en distintas actividades de formación de tales establecimientos, se propone un nuevo análisis de la noción de inclusión, centrado no en la orientación sexual de los sujetos -como las escuelas la suelen representar-, sino en la construcción de la alteridad que cada currículo conlleva. Como resultado de esta investigación, se evidencia la dificultad para comprender los límites que la experiencia subjetiva impone a la discusión moral sobre sexualidad. En estas escuelas católicas las formas de inclusión siguen estando confinadas a compartir una misma posición socioeconómica, sin comprender como persisten ciertas alteridades invisibles cuya inclusión probablemente evidenciaría el carácter contingente de la educación sexual.
\end{abstract}

Descriptores: Diferencia individual; Estratificación social; Educación sexual; Escuela católica; Sexualidad.

In elitist Catholic schools, sex education attempts to regulate intimacy and personal character, in order to shape an individual capable of guiding himself and his sexuality towards an ethical project. In this article, we will examine the result of a qualitative research carried out in 16 Catholic establishments in the city of Santiago. From 53 interviews with counselors and psychologists in charge of sexual education, two focus groups that convened 23 adolescents from these schools and ethnographic observations in different training activities of such establishments, a new analysis of the notion of inclusion is proposed. In this case, we are not focused on the sexual orientation of the subjects - as schools usually do, representing the idea of sexual diversity- but instead, we will observe the construction of the otherness associated to sexual education. As a result, the limits that subjective experience imposes on moral discussion about sexuality become evident. In these schools, "diversity" is still confined to share the same socioeconomic position. The inclusion of certain invisible alterities would probably evidence the contingent nature of sex education.

Keywords: Individual differences; Social stratification; Sexual education; Catholic school; Sexuality.

*Contacto: pastudil@uahurtado.cl

ISSN: 0718-7378

www.rinace.net/rlei/
Recibido: 25/10/2019

$1^{\text {a }}$ Evaluación: 23/11/2029

Aceptado: $\quad 10 / 01 / 2019$ 


\section{Introducción}

Se podría pensar que una educación sexual inclusiva es aquella que incorpora contenidos propios de las identidades LGBTI, identificadas con la noción de "diversidad sexual". No obstante, la inclusión sería parcial si se reduce únicamente a una incorporación de lo que está en los márgenes. Cuando se habla de educación sexual inclusiva lo relevante es observar el modo cómo se incorporan las experiencias subjetivas, vivencias que están delimitadas no solo por la orientación sexual sino por una serie de otros elementos relacionados con la propia posición social.

Lo anterior es aún más relevante cuando en Chile, la educación sexual se orienta sobre la idea de formar un sujeto que toma decisiones de manera informada, sin que exista un único foco que asegure que dicha educación será equivalente para todos los individuos del país (Figueroa, 2012; Palma, Reyes y Moreno, 2013). En el caso de los colegios católicos los programas de educación sexual intentan resolver permanentemente la tensión entre reglas del magisterio que apuntan a la abstinencia prematrimonial y las normas de la cultura contemporánea que diversifican las posibles trayectorias sexuales de los individuos (Bozon, 2009; Montero-Ossandón, Valverde-Forttes, Dois-Castellón, Bicocca-Gino, Domínguez-Hidalgo, 2017). Esta discusión de carácter más bien moral impide observar los límites de inclusión que dichos programas terminan por producir y reproducir.

Actualmente, en las escuelas católicas de élite no todas las experiencias de los sujetos tienen cabida. Y no nos referimos aquí a las diferencias que suponen las distintas orientaciones sexuales o expresiones de género. En aquellas, los debates en torno a la sexualidad adquieren ribetes más bien morales, sin problematizar el modo como las normas sexuales son socialmente producidas y tienen un efecto en el reconocimiento de los sujetos (Astudillo, 2016; Maturana et al., 2016). Como veremos a continuación, existen límites y fronteras que distinguen a los individuos con relación a como entienden la sexualidad, algunas de las cuales, al ser pasadas por alto, impiden comprender el carácter contingente de la sexualidad y avanzar en la inclusión de las distintas formas de vivirla en distintos espacios sociales.

\section{Marco teórico}

Como cualquier proyecto educativo, la educación sexual implica formar un individuo para acercarlo a un ideal de sujeto determinado (Dubet y Martuccelli, 1996). En Occidente, este ideal de sujeto sexuado se entendería a partir de una idea de autonomía donde los individuos deben hacerse cargo de su afectividad y sexualidad de modo relativamente aislado y personal (Bozon, 2009; Giddens, 2006; Illouz, 2015). Es por esta razón que los programas tienden a insistir en la idea de entregar información para que los sujetos tomen decisiones personales responsables (Figueroa, 2012).

Ahora bien, esta norma social supone reconocer la agencia de todos los sujetos en relación con su sexualidad, algo que entra en tensión con el posicionamiento cotidiano de los sujetos. En otras palabras, si bien se espera que todos los individuos se hagan cargo de sexualidad no todas las sexualidades individuales son reconocidas de la misma manera. Como sostienen Gayle Rubin (1984) y Judith Butler (2018), en Occidente, las identidades personales tienden a ser elaboradas a partir de una norma de sexo/género que favorece a los hombres heterosexuales y subordina todas las otras posibles combinaciones, entendidas de modo ontológico y no como resultado de una producción política. Ahora 
bien, como lo advierte el análisis interseccional esta desigualdad se entrecruza también con otras categorías identitarias: la clase social, la raza o la adscripción étnica por nombrar algunas (Collins y Bilge, 2016).

Esta clase de diferencia puede tener un efecto estructurante en las representaciones y experiencias sexuales. Por una parte, porque las diferencias producen asimetrías. Phil Hubbard (2002) señala que la sexualidad -sea a partir de un encuentro real, sea a partir de una fantasía- supone siempre el encuentro de dos individuos que son definidos como un Alter y Ego el uno para el otro. Ahora bien, dicho reconocimiento difícilmente opera sobre un marco de igualdad, pues los individuos están adscritos a determinados grupos con diferentes posiciones en la vida social. Como señala Eva Illouz (2015), la sexualidad es una forma de poner en acto las jerarquías sociales y la moralidad de una sociedad. La experiencia individual, de este modo, nunca está completamente aislada del modo como la sexualidad y la idea de un sujeto sexuado son producidas socialmente.

Por otra parte, las diferencias se entienden en un marco de complejidad. A la sumatoria de categorías que ya hemos identificado se debe sumar el hecho que la identificación subjetiva con tales variables es de por si inestable. En el contex to de sociedades modernas cada vez más fragmentadas y diferenciadas, los individuos responden a cierta fluidez identitaria, donde constantemente toman distancia de formas heredadas de pertenencia como la comunidad, la familia, la nación, la clase o el género, para reivindicar su derecho a elegir pertenecer o no a determinados grupos (Singly, 2010). En este sentido, el análisis interseccional, debería incorporar además la manera como el sujeto se posiciona frente a cada uno marcadores sociales de la diferencia. Esto, porque los individuos no solo son capaces de explicar que significa una categoría, sino que además pueden ofrecer explicaciones respecto a cómo los demás sujetos se comportan en virtud de ellas (Dubet, 2010, 2013).

A modo de ejemplo, el ser mujer de una clase acomodada puede implicar estar sujeto a una socialización específica de género -con consecuencias sobre el comportamiento sexual esperado- pero no necesariamente esto significa que la persona en cuestión entenderá sus vivencias a partir de dicha suma de posiciones (género y clase). En ocasiones, intentará renunciar a este posicionamiento en vista de sentirse "igual a otros" porque esa es la norma dominante en una interacción dada. Por tal razón, es importante reflexionar sobre el carácter contingente y situado de las normas en materia de sexualidad. Como hemos visto, la identificación con determinado grupo conlleva ciertas prerrogativas, las cuales son construidas a partir del silenciamiento y/o subordinación de otras experiencias, pero no necesariamente esa cartografía será dominante al momento de narrar la realidad.

Como lo señala Stauntson (2019), la identidad personal siempre se construye confrontando lo que uno es con aquello que no se es, reconocimiento que a su vez depende de una cartografía social de diferencias válidas y otras que son descartadas. Por tal razón no se pueden comprender las narrativas sobre la sexualidad sin reconocer como los sujetos describen la diferencia. Volviendo sobre Hubbard, la relación entre Ego y Alter depende de una cartografía de reconocimiento que es a la vez compleja y dinámica. Habiendo comprendido lo anterior, podemos mirar de una nueva forma el modo como se educa la sexualidad en la escuela.

En este artículo tomaremos como caso de análisis la educación sexual de los colegios católicos de élite. En ellos, el proyecto educativo se caracteriza por su pretensión de formar un sujeto capaz de gobernarse a sí mismo conforme al seguimiento de una vocación divina 
(Thumala, 2007). En materia de sexualidad, esto refleja la base personalista del magisterio eclesial que opera sobre la idea que un individuo debe autónoma y responsablemente orientar su sexualidad hacia una donación de sí (Montero-Ossandón, 2017). Estas ideas de sexualidad -movilizadas por el currículo y las prácticas cotidianas de estos establecimientos- necesariamente dan pie a formas de posicionamiento personal y narrativas sobre la diferencia.

La posición de Ego no se entiende sino es también a partir de lo que define al Alter. Si la sexualidad es construida políticamente en el marco de sociedades diferenciadas que ponen el acento en la individualidad, la diversidad de experiencias es prácticamente inabarcable. Luego, para comprender los límites de la diversidad en la educación sexual de los colegios católicos es necesario poner en tensión dos elementos que se relacionan entre sí: por una parte, el modo como se recrea la diferencia (como se reconoce al Alter); y por otra, la manera como la propia posición impide observar los límites de esa misma diferencia (como hay ciertas alteridades que no son reconocidas). Teniendo en cuenta ambos elementos se puede comprender como la noción de diferencia permite comprender la diversidad de un modo distinto hasta como ahora se ha entendido.

\section{Método}

El presente artículo se enmarca en la investigación doctoral titulada "Tensiones y paradojas de un proceso de individuación sexual. El caso de las escuelas católicas de élite en Santiago de Chile" cuyo trabajo de campo se desarrolló en Santiago de Chile entre mayo de 2014 y diciembre de 2015 en colegios que atienden a familias de altos ingresos de la ciudad. La selección de establecimientos se realizó a partir de diferentes contactos que fueron facilitados entre los mismos entrevistados a la manera de un muestreo "bola de nieve" lo que permitió representar un total de 13 establecimientos dependientes de distintas congregaciones y movimientos católicos, cuyos proyectos educativos muestran diferentes sellos institucionales, además de 3 escuelas laicas que atienden al mismo grupo socioeconómico.

En relación con este artículo la información es obtenida a partir de 39 entrevistas en profundidad realizadas ante adultos, hombres y mujeres de entre 32 y 56 años, quienes describieron sus responsabilidades en la conducción de la educación sexual de sus establecimientos; y de dos grupos focales realizados con estudiantes de dos de los colegios de la muestra. Estos grupos reunieron un total de 23 participantes entre 15 y 16 años quienes opinaron respecto de la formación en sexualidad que recibían en sus escuelas. En paralelo, se efectuaron dos observaciones participantes en actividades de voluntariado de esas mismas instituciones (trabajos de verano e invierno), las cuales fueron referidas como un importante componente del currículo escolar por parte de los entrevistados.

La información obtenida en las entrevistas y grupos focales fue tratada mediante una metodología de análisis del discurso. Para ello, se recupera la noción de experiencia que propone Joan Scott (1991) donde se asume que las categorías con las que un sujeto entiende y narra la realidad son el resultado de una construcción social históricamente situada. Lo anterior, permite mirar de manera crítica el posicionamiento de los actores respecto a las normas de sexualidad movilizadas en la educación sexual. Para el caso de los grupos focales, se recogen además las advertencias de Manuel Canales (2013) respecto a las relaciones entre la palabra personal y la palabra del grupo, donde las experiencias 
narradas solo se relatan apelando a un sentido común, un fondo compartido de distinciones y suposiciones. Como consecuencia, se puede acceder a aquellos nudos críticos del posicionamiento individual a partir de la descripción de las normas comunes, de los ejemplos y anécdotas compartidas o de los juicios morales sobre las reglas compartidas de un grupo, resaltando de paso el protagonismo de los adolescentes en su propio proceso de educación sexual.

Algo similar ocurre con el tratamiento analítico de las anécdotas surgidas de las observaciones participantes, pues aquellas condensan diferentes normas sociales que se hacen visibles a partir de su reproducción o de su transgresión. En el contexto de este artículo, los registros etnográficos adquieren el valor de evidencias, pues permiten contrastar las narrativas estudiantiles con situaciones concretas de interacción social donde aquellas normas se cumplen o se resisten, movilizando emociones como también sentidos anclados socio-históricamente (Bericat, 2000).

\title{
3. Resultados
}

\subsection{Las alteridades reconocidas: una diversidad de tipo moral}

La educación sexual no escapa a la lógica de reconocimiento que caracteriza a los procesos de construcción identitaria contemporáneos (Singly, 2010) Esto queda claro tempranamente, al observar cómo los estudiantes reconocen sus propias opiniones, exigiendo que aquellas sean, al menos, discutidas con sus educadores.

\begin{abstract}
Encuentro que la educación sexual en tercero y cuarto medio debiese ser retomar esto de los métodos anticonceptivos, las enfermedades de transmisión sexual, cómo evitamos, qué hacer, qué no hacer, dónde es peligroso, dónde no, o sea, ese tipo de cosas. Igual encuentro que son útiles las clases, pero para ser sincera escucho la mitad. De repente dicen cosas que me han hecho pensar o de repente dicen cosas con las que tú estás completamente en desacuerdo. De repente hay peleas con profesores que se tienen que bancar la discusión. No sé, creo que hay que abrir más espacios de diálogo. Sé que estamos en un colegio católico, pero necesitamos que los profes se abran a escuchar cosas nuevas. En el fondo no vale de nada hablar de moralidad constantemente si nosotros no estamos de acuerdo, porque también debe ir cambiando la moralidad, o sea, la moral siempre cambia, depende de la sociedad, en qué sistema estamos inmersos, entonces algo que se aplica hace muchos años es algo que no es aplica hoy en día. Y la orientación sexual debe empezar desde más chicos porque las jóvenes hoy en día están hardcore. (Estudiante, mujer, grupo focal 2 )

Encuentro que nos debieran enseñar aparte de los valores del colegio el cómo prevenir el embarazo para después no tener que abortar, o el cómo si es que vas a perder la virginidad saber con quién, el cómo, el dónde, porque a veces importa mucho el lugar... un poco más de la vida real que está pasando hoy en día, más que los valores del colegio impregnados en un texto / E5: Si, porque todo el tiempo nos dicen 'y cuando estén casados, no sé qué' y no asumen que más del $90 \%$ de los chilenos no llegan vírgenes al matrimonio. Entonces como que eluden la realidad. En los colegios de Estados Unidos, yo he visto películas por lo menos, los trauman poniéndoles las enfermedades de transmisión sexual, los dejan traumados como con imágenes y videos I Igual a nosotros nunca nos han dicho como que "el sexo es malo" nunca, nunca. Pero asumen que una va a llegar virgen al matrimonio y eso no es cierto. (Estudiantes, hombre y mujer, grupo focal 1)
\end{abstract}

Podemos ver aquí como los estudiantes buscan reivindicar una disposición activa frente a su propia educación sexual, y al mismo tiempo, cómo moviliza una distinción generacional que sostiene este posicionamiento personal. Recuperamos acá la distinción entre Ego y Alter encarnado en este caso por la experiencia propia y la de los actores que tratan de 
imponer otra realidad. Por una parte, los estudiantes se posicionan moralmente reflejando la tensión inherente a las escuelas católicas, donde la educación sexual intenta conciliar tanto la norma magisterial como la lectura de la cultura contemporánea, cuestión visible en su intención de integrar puntos de vista diferentes a los de la Iglesia, para aproximarse más bien a la "realidad actual".

En paralelo, los adolescentes articulan categorías de identidad en virtud de como los grupos defienden o encarnan una u otra norma, algo que a su juicio estaría fuertemente asociada las diferencias generacionales. La posición crítica se entiende en comparación a ciertos profesores, pero también -como lo muestra la primera cita- en relación con alumnos de cursos menores. Esta primera forma de posicionamiento personal -la definición de Ego- se hace sobre la base de una idea de pertenencia y distinción específica, pero no única. Cuando se discute con los estudiantes sobre su educación sexual surgen de inmediato nuevos tipos de diferencias que van complejizando la cartografía de posiciones posibles.

La segunda semana de vacaciones vino una amiga mía que es de Argentina. Lo que
más llamó la atención a mis amigos chilenos es que la vieron súper liberal. De repente
ella estaba hablando de la masturbación: ¿ustedes se masturban? y todas mis amigas:
¡no, no, te juro que no! y ella "Ustedes son unas mentirosas". Como que el chileno se
cohíbe mucho, le da mucha vergüenza, no sé, masturbarse, no sé. Y después ella
comentaba “mi mejor amigo se ha agarrado a dos huevones" y si acá un amigo se
agarra a dos huevones erís gay. Comparativamente, el chileno se cohíbe mucho.
(Estudiante, hombre, grupo focal 2)
Resulta que ahora todas las mujeres en las fiestas están apoyadas contra la pared para
que el hombre las puntee por detrás. O sea, yo recuerdo que yo bailaba y en ningún
momento me puntearon de esa manera. Por eso yo quedé bastante asombrada cuando
vi toda la pared ocupada, minas pegadas a la pared, poniendo el poto para que las
puntearan. Ahora todo se empieza antes. Es más fácil conseguir alcohol, conseguir
drogas, tener sexo que antes. Como que todo está a la mano, entonces lo aprovechan,
he visto niñas de doce años tomando o niños de doce que ya perdieron la virginidady
una aquí de dieciocho (años) completita. Son cosas que pasan. (Estudiante, mujer,
grupo focal 2)

Vemos aquí como los individuos pueden tomar distancia de las normas encarnadas también por sus pares. Tanto al imaginar la "realidad" de otro país como en la crítica con otras adolescentes, los estudiantes movilizan una manera de entenderse a sí mismos. En ninguna de las dos citas la comparación expresa un deseo de "ser como esos otros" sino más bien evidencia una autoafirmación de cómo soy/somos.

Un tercer tipo de diferencias se hace visible en la sospecha que opera frente a los individuos conservadores. El autorreconocimiento de los estudiantes como agentes de su propia educación sexual -y por ende de su sexualidad- supone una devaluación de cualquier posición acrítica y una sospecha frente a los individuos que la encarnan.

Seguramente muchos de esos cruzados del Opus Dei que ven la vida con tanta regla, al final se cuestionan / E2: Claro, que se cuestionan. No sé, te los encuentras y pueden ser los más borrachos o los que están más vueltos locos en la fiesta ¿̇Por qué? No lo sé bien, pero me imagino que viven siempre con mucha regla y al final eso no ayuda mucho. Lo mismo que los castigos, no sé, cuando uno es más chico. / E1: Es como lo lógico, si te castigan tanto y lo ponen tan así, obviamente que como adolescentes quieren hacer lo contrario, están tan chatos de que esté todo tan restringido que después salen a la vida y los tienes ahí, desatados... (Estudiantes, hombres, grupo focal 1)

Ella (la profesora) es muy joven, sabe mucho más y te da más confianza porque es alguien que cuenta sus cosas. No sé, a nosotros nos ha pasado casos de profesores que no nos tiene confianza y no nos cuentan nada de su vida. Como que se cacha altiro que no se puede hablar mucho con esos profesores que son del movimiento (se refiere a 
una agrupación religiosa asociada a su colegio) porque son profesores que son demasiado llevados a sus valores y no van a aceptar otra cosa. $Y$ a nosotros nos importa que sean más abiertos de mente y que acepten nuestra realidad, no sé, alguien que a la hora de almuerzo le podamos contar de nuestro fin de semana. Esta profesora joven no es como los otros, porque ella es de la que te dicen: 'si van a tomar tienen que comer antes, yo lo aprendí de chica' y eso como que da mucha confianza. (Estudiante, mujer, grupo focal 1)

Las normas movilizadas por la educación sexual religiosa acá son leídas según sus efectos estructurantes en los sujetos, más que por su contenido normativo propiamente tal. Para estos estudiantes, lo relevante es interrogarse si acaso la regla conservadora regula la superficie o la interioridad del sujeto, porque la norma por sí misma solo adquiere valor si es capaz de transformar la subjetividad individual. Tal como lo señala François Dubet (2010), los individuos son siempre capaces de desarrollar formas de aceptación y distancia estratégicas frente a las normas que intentan regular su conducta, cuestión que, podemos suponer, también se extiende a otros a quienes se juzga como agentes de su propio comportamiento.

Quizás esta es la razón que explica el por qué, independientemente del enfoque moral o de los contenidos efectivamente trabajados por la educación sexual de las escuelas analizadas, ningún sujeto reivindique una postura conservadora en sus programas. El peso de este último criterio de diferenciación moral es tal, que, para los equipos psicosociales en las escuelas, el conservadurismo siempre está ubicado "más allá" del propio establecimiento. Esto permite afirmar consistentemente que "uno no es así de conservador como los otros".

\begin{abstract}
Nosotros tratamos de erradicar esta visión de que el sexo es una cosa sucia, mala... ¡lejos de nosotros toda esa idea! Por todos los medios tratamos de que no nos vean así. Yo creo que esa visión de la sexualidad así mala, negativa, bueno primero no es católica, nunca la ha sido, yo creo que es una visión bastante más protestante, puritana, de lo que está muy teñido el cine, las series de televisión. Entonces yo creo que eso nos falta, que cuando nosotros vemos que en la televisión o el cine se hace referencia a la visión cristiana de la sexualidad y ellos la muestran, así como una cosa represiva llena de culpas, nosotros deberíamos decir que esa es una visión puritana, que de católica no tiene nada. (Encargado de educación sexual, colegio católico)
\end{abstract}

Vemos hasta aquí como, al hablar sobre sexualidad y educación sexual, los jóvenes van produciendo y reproduciendo una idea de sujeto, a partir de ciertas formas de diferenciación social. Sin embargo, las formas que adopta la diferencia no son siempre visibles para los sujetos. Las alteridades silenciadas son, en este sentido, una frontera no reflexiva para la inclusión. A partir del ejemplo concreto de cómo se representa el género, como las experiencias de otros contextos sociales no logran permear la cartografía de la diversidad cuando se habla de sexualidad en los colegios católicos.

\title{
3.2. La alteridad no reconocida: la frontera social
}

Las distinciones que acabamos de ver son siempre contingentes y variables. Las diferenciaciones entre Alter y Ego, específicas de un espacio social, podrían siempre cristalizarse de otra manera en un contexto diferente (Connell y Pearse, 2018). Para ilustrarlo, examinaremos cómo las ideas de sexualidad y de género no son independientes de la pertenencia a un grupo socioeconómico determinado.

Tomemos en consideración la evidencia recogida durante las observaciones etnográficas realizadas en los trabajos de verano de algunos de los colegios que formaron parte de este estudio. Como suele ocurrir en este tipo de actividades, un centenar de estudiantes más quince adultos se instala durante una semana en una escuela de un barrio vulnerable, para 
desde allí prestar servicios a la comunidad (reparación de casas, trabajos de animación infantil, etc.) Durante todo ese tiempo, hombres y mujeres alojan en habitaciones separadas y utilizan baños también separados por sexo. En este contexto ocurre la siguiente anotación:

Día 4: 23.15 hrs. Estoy cepillándome los dientes en el baño de hombres, justo antes de irme a dormir y que se haga el llamado a silencio dentro de la escuela. Las instalaciones muestran la suciedad y desorden propios del final del día y el uso intensivo antes de la acostada. Al entrar en la zona de los lavamanos puedo comprobar que en los espejos han sido dispuestos unos post-it con algunos mensajes que buscaban -supongo- amenizar la experiencia y personalizar el espacio. Probablemente habian sido dejados por la cuadrilla que esa mañana había limpiado los baños. Puedo leer pequeñas frases que tienen por objeto subir la autoestima de los varones que ocupábamos ese lugar (figura 1). Intrigado, me pregunto si en el baño de las mujeres habría algo parecido. Una vez afuera, le pido a una de las estudiantes con las que trabajé en la mañana que me hiciera el favor de tomarles fotografías con mi teléfono móvil a los mensajes que pudiera haber en el baño de mujeres. Después de 3 minutos compruebo que alli también había post-it, pero que la naturaleza de los papeles pegados era completamente distinta: mientras a ellas se les daban consejos para cuidar su aspecto (figura 2), a nosotros se nos hablaba del deseo que podíamos provocar en las mujeres, sin tener que hacer nada para atender nuestra apariencia. (Anotación cuaderno de campo. Martes 6 de enero de 2015)

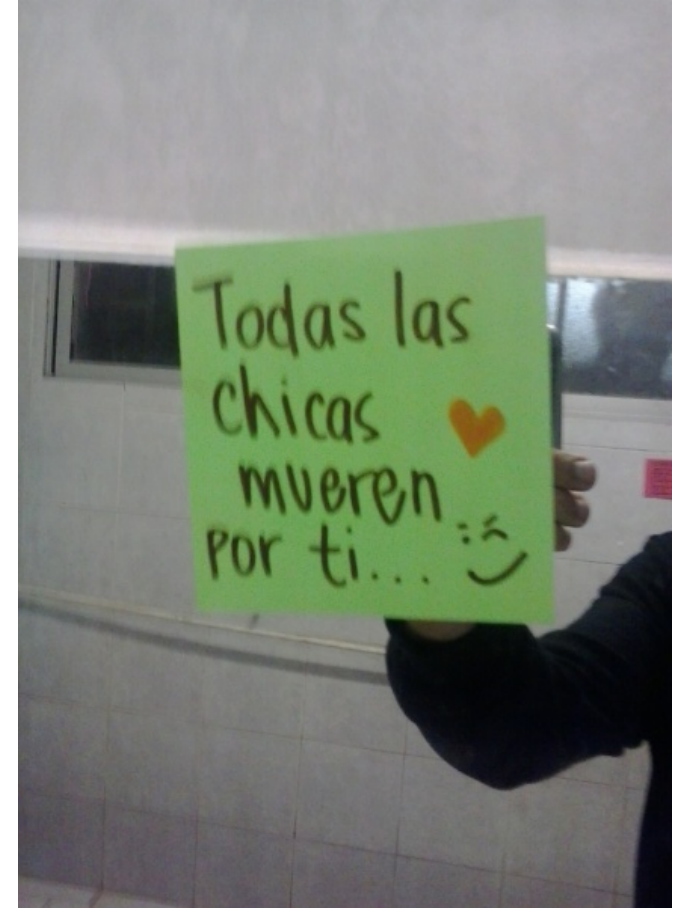

Figura 1: Espejo del baño de hombres Fuente: Elaboración propia.

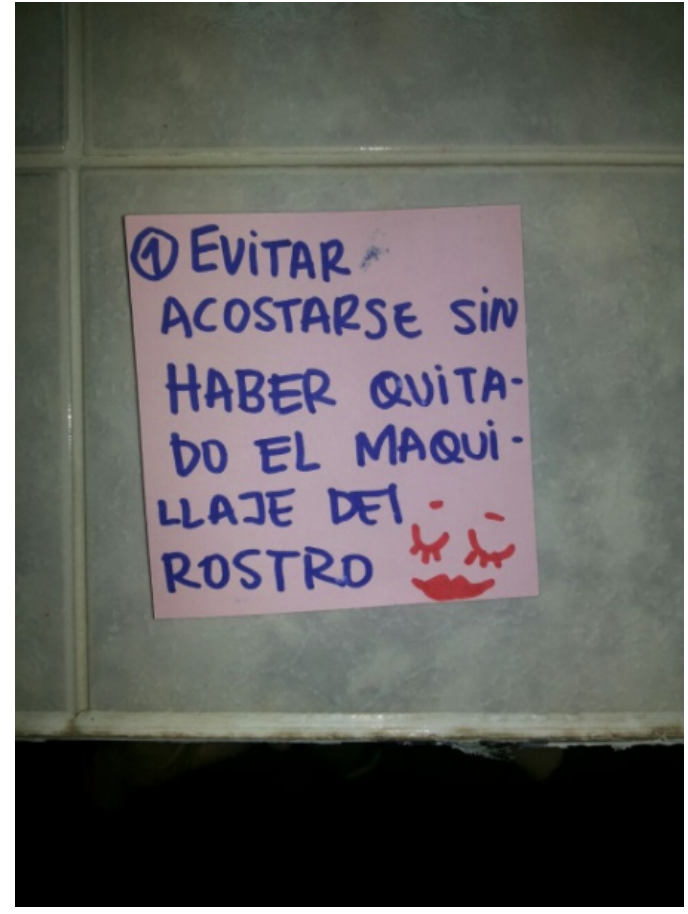

Figura 2: Espejo del baño de mujeres Fuente: Elaboración propia.

A priori, este registro de campo parece una evidencia más del ambiente distendido característico de estas actividades formativas. Sin embargo, es claro como los post-it transmiten también mensajes organizados en virtud de un esquema tradicional de diferenciación de género. Esto es algo que se hace evidente en un segundo ejemplo. Durante varias tardes, se acostumbraba a leer los mensajes anónimos que cualquiera podía dejar en un buzón a lo largo del día. Constantemente los mensajes tenían un carácter 
humorístico centrado en dos cosas: la falta de astucia de las mujeres y la compulsión masturbatoria de los hombres. En ambos casos el sexismo es evidente. Los estudiantes, al ser entrevistados, evidencian una crítica respecto a cómo los adultos entienden la diferencia entre hombres y mujeres y, sin embargo, no formulan críticas abiertas a las disposiciones que ellos reproducen, no al menos cuando los trabajos voluntarios tienen lugar. A pesar de no estar escrito, la distinción de género subsiste en varios componentes de la actividad: el trabajo que cada persona podía hacer (pese a la insistencia que "las mujeres igual pueden martillar"), el modo como se ocupaba el espacio común de la cancha, la prohibición de que una pareja de pololos (novios) conviviera en la misma escuela, la vigilancia sobre la ropa que vestían para trabajar las mujeres, pero no los hombres, por nombrar algunos.

Probablemente lo que explica esta anulación de la crítica sea el fuerte carácter comunitario de la actividad (Thumala, 2007), donde los estudiantes se reconocen como parte de un colectivo y de un proyecto educativo escolar. A diferencia de lo que vimos en el apartado anterior, este tipo de posicionamiento no es moral, sino social. Es este cambio de foco lo que permite dilucidar mejor las fronteras del sujeto sexuado. Cuando otras formas de alteridad aparecen en el propio espacio social muchas veces no hay repertorio para leer las diferencias.

Día 2. 04.0o hrs. Estaba durmiendo cuando me despierto con el ruido de las piedras que, desde la calle, caían sobre el techo de la escuela. Como puedo, me incorporo, pero nadie enciende la luz y no logro darme cuenta si alguien más se ha despertado. Escucho a quienes, asumo, eran jóvenes del sector que querían llamar la atención de las mujeres que dormían en las salas contiguas a la mía. Escucho frases del tipo: "qué salgan las minas ricas", "que se asomen las rucias", "les vamos a chupar las tetas", "seguro tienen la zorra rica". Cinco minutos más tarde el grupo se retira y el ruido cesa. La sala sigue en silencio por lo que supongo que el resto del grupo duerme y me vuelvo a dormir.

o8:30 hrs. Estoy en el comedor tomando desayuno. Me levanté pensando que el "evento" sería el comentario obligado de la mañana. Comparto la mesa con asesoras que habían dormido en la pieza al lado de la nuestra. Nadie hace comentario alguno sobre el hecho. Llego incluso a dudar que el apedreo hubiera efectivamente ocurrido.

22:00 hrs. Reunión de asesores. Hay presencia de directivos del colegio, quienes han venido de visita. El jefe del campamento comenta "me imagino que escucharon lo que pasó anoche ¿̇no?”. Algunas asesoras reconocen que habian despertado y que durante el día se fueron enterando de que algunas estudiantes también habian escuchado los piedrazos y los gritos. El jefe del campamento indica entonces: "bien, si esto vuelve a pasar es mejor no comentarlo demasiado, de otro modo las mujeres se pueden poner nerviosas y no vale la pena agrandar la cuestión”. El grupo asiente y se cierra el tema. Nadie vuelve a comentar algo al respecto por el resto de la reunión. (Anotación cuaderno de campo, domingo 4 de enero de 2015)

A nuestro juicio, esta anécdota resulta particularmente ilustrativa de los límites del sujeto sexuado. Lo que el jefe de trabajos resuelve no solo evidencia un sexismo implícito -solo se podían poner nerviosas las mujeres y no los hombres, o la única reacción esperable por parte de las mujeres era el nerviosismo- sino que también silencia una forma de sexualización de las relaciones que, en esta ocasión, proviene desde fuera del grupo. Nada garantizaba que los jóvenes y adolescentes que vivían en el barrio no fueran a reproducir los "piropos" durante el resto de la semana y nadie se preguntaba si realmente se podía pasar de la insinuación grosera a un acto concreto. La connotación sexual que había tenido el apedreo en cuestión parecía insignificante, comparada con otras formas de regular la sexualidad esa misma semana. 
Lo que esta anécdota muestra es la importancia de ciertos límites sociales para incorporar la sexualidad al análisis de una interacción cualquiera. De cierta manera, lo grosero del piropo era quizás la mejor prueba de que los jóvenes nunca les iban realmente a "chupar las tetas a las rucias" que venían a trabajar, sin embargo, es innegable que el deseo erótico no se agotaba en el apedreo a la escuela. La frontera social que vuelve invisible tal deseo dentro de la convivencia de los trabajos de verano nunca es problematizada.

En estos colegios, la experiencia del voluntariado está llena de anécdotas del mismo tipo, donde los adolescentes pueden entrar en contacto con la intimidad sexualizada de otros individuos sin que esta sea leída de modo sexual.

En nuestros voluntariados a veces encontramos travestis. $\Upsilon$ estas niñitas son muy mujercitas, muy femeninas. $\Upsilon$ pasó alguna vez que algunas de ellas les empezaron a decir 'bueno, si yo tuviera pasarela caminaría así y cómo lo harías tú. Y el travesti empieza a como exacerbar sus modales y ellas se matan de la risa de él. Entonces me tocó decirles después en el viaje de regreso 'oye no es la forma de tratar a las personas, porque en el fondo tú no la estás respetando, le estás faltando el respeto a su condición de gay'. De repente una tiene que enrielarlas, hacerles ver los temas de conversación que pueden tener con la gente, porque de repente no tienen criterio para comparar su realidad con las de ellos. No quiere decir que las alumnas no sean auténticas, que no sean ellas mismas, sino que les falta ser más empáticas, ponerse en la situación de ellos. (Orientadora, colegio católico)

Nosotros tenemos mucho cuidado y hablamos mucho con ellos, cuando nos enfrentamos a esa situación de que hay adolescentes del lugar que vienen a vernos. Puros niños rubiecitos que van a estar acá cinco días y entonces empiezan a rondarlos, digamos. Nosotros hablamos [con nuestros estudiantes para] que el contacto sea mínimo y siempre con respeto y muy educados, que nadie diga nada, tampoco meterles conversa porque para qué, digamos (...) Que yo sepa nunca ha pasado nada, aunque veo que esas mujeres siempre tratar de establecer una relación y yo me quedo bastante sorprendido de los caballeros que han sido nuestros estudiantes, porque no son tan caballeros en la vida diaria. Porque en una fiesta ellos se agarran [mujeres], pero no en estos viajes. Nosotros los aconsejamos bastante también, llega hasta ahí no más o va a venir el pueblo entero a pegarte. (Profesional, colegio católico)

La asimetría entre individuos resulta evidente. La sexualidad de los otros (travestis, adolescentes del lugar) no interpela la propia vivencia de la sexualidad, cruzada en este caso por la posición social. La posibilidad de establecer una reflexión crítica es limitada. Ahora bien, la clave de dicha invisibilidad parece residir en la dificultad para reconocer las desigualdades sociales. Tal como lo reconoce un psicólogo a cargo de los programas de educación sexual de uno de los establecimientos analizados, resulta muy difícil hacer visible este límite crítico, pues hay una resistencia a reconocer los propios privilegios:

En general, yo te diría que no hay contacto entre los alumnos de los dos colegios (...) en general hay mucha cortesía entre los estudiantes, pero no sé si se cruzan realmente. Es como si estuvieran hablando desde la puerta de la casa, ninguno los deja entrar de verdad a su casa. Sin embargo, tú vas a escuchar que nuestros alumnos tienen un discurso súper igualitario, anulador de las diferencias de clases sociales. Nuestros estudiantes van a decir que no somos un colegio cuico, porque lo peor que tú puedes decirles a los alumnos de este colegio es que son cuicos, que son clasistas. Siempre te van a decir que los cuicos van a otros colegios, que nosotros en cambio somos un colegio diverso. Pero no sé cuán diversos son, en términos socioculturales o sociales, no sé cuán diversos son. (Psicólogo, colegio católico)

Aquí la frontera social que puede ser vista, pero difícilmente puede ser reconocida. 


\section{Discusión}

Todas las fronteras que reconocen y no reconocen los estudiantes, y otros actores en la escuela, permiten vislumbrar el carácter socialmente construido de la sexualidad. La experiencia de esta es entendida siempre dentro de marcos que son contingentes y moldean un límite para su comprensión y relato.

Tal como lo señala el análisis interseccional el comportamiento sexual no puede ser entendido fuera del marco que produce el género, la raza o la clase social (Collins y Bilge, 2016). Vemos en los resultados anteriores como el encuentro entre ambos grupos socioeconómicos haría evidente que las narrativas sobre la sexualidad responden también a condiciones específicas de individuación y, por ende, de construcción de la subjetividad.

En virtud de reconocer la subjetividad de los agentes, debemos admitir la premisa que los estudiantes activamente desean no ser clasistas. La pregunta entonces es saber cuándo la clase es reconocida, en el caso de qué interacción. De acuerdo con la evidencia recogida, ningún programa de educación sexual aborda reflexivamente la existencia de diferencias sociales en lo que refiere a la sexualidad, algo que es consistente con la formulación de la política pública sobre educación sexual (Morgade, 2016). La preparación para la vida sexual y afectiva - controlada por los adultos- se entiende siempre en el marco de un proyecto de pareja cuya composición nunca se tematiza desde el punto de vista social, pese a que la evidencia muestra que la estabilidad de los proyectos contemporáneos descansa en un principio de reproducción social heteronormada que es reforzado por los mismos programas de educación sexual (Le Mat, 2016: Maturana et al., 2016)

Tal como lo evidencia Madrid (2016) a propósito del chaneo, en Chile la sexualidad masculina se estructura también a partir de la capacidad que tienen los sujetos de cruzar la barrera socioeconómica entre los grupos, para acceder a experiencias sexuales con mujeres de una extracción social más baja, las cuales nunca pueden estabilizarse en un pololeo (noviazgo). La distinción de clase en este caso sirve para organizar el repertorio de conductas y expectativas. Llama la atención en este sentido que escuelas que organizan su formación en torno a la idea de un individuo con conciencia social (Thumala, 2007) no incorporen una discusión en torno a esta práctica que los involucra directamente. Lo anterior es significativo toda vez que la educación sexual inclusiva debiera ser capaz de reflexionar en torno a las relaciones de poder que también caracterizan a la sexualidad adolescente. Sistemáticamente, la educación sexual opera sobre la idea de un sujeto neutro carente de poder personal, perspectiva propia del adultocentrismo (Amsellem-Mainguy y Vuattoux, 2019)

En las escuelas chilenas, la idea de inclusión sexual se ha entendido más bien limitada a la noción de diversidad sexual. La política pública crecientemente promueve el tratamiento respetuoso y afirmativo de la orientación sexual, identidad y expresión de género de los sujetos, sin considerar otras variables que pudieran afectar la posición de reconocimiento de los individuos en la escuela, como podría ser la clase social y las condiciones materiales que permiten su reconocimiento (Rojas et al., 2019) y especialmente la autoafirmación que nunca es independiente de como en una escuela los sujetos posicionan las diferencias y los sujetos que las encarnan (Miller, 2016). Vemos en los resultados que la referencia a otros -los conservadores, los adolescentes de otros países, las niñas hipersexualizados- termina por reforzar una idea de diferencia que es limitada en términos sociológicos. La relación entre Alter y Ego se sostiene sobre una capa de experiencia compartida que se articula 
sobre prácticas cotidianas que reproducen formas de estratificación social (Bourdieu, 2012). Esto supone prescindir de cualquier reflexión sobre los límites sociales que separan grupos y que configuran la "propia realidad", aun si la misma está permanentemente ligada a condiciones materiales que permiten las distintas experiencias (Cover, 2019), condiciones que difícilmente son tematizadas en los programas de educación sexual (Márquez, Gutierrez-Barroso, Gómez-Galdona; 2017).

En el caso que examinamos esta clausura material es la que sostiene la abstracción del sujeto detrás de la educación sexual católica de élite. Desde este punto de vista, una educación sexual inclusiva requeriría integrar el modo como cada actor es reconocido como un individuo sexuado y, por lo tanto, capaz de encarnar o no ese ideal de sujeto detrás del proyecto educativo, algo que las escuelas constantemente alimentan a través de la reproducción de prejuicios (Barrientos et al., 2019; Staunton, 2019). En este caso, lo que incorporamos al análisis es la invisibilidad de algunas diferencias. Las diferencias morales refuerzan un sentido de pertenencia que deja fuera lo social.

\section{Conclusión}

Cuando se examina la educación sexual de los colegios católicos de élite, se comprueba rápidamente que dicho currículo produce determinadas posiciones morales que también se relacionan con posiciones sociales. Los adolescentes de los colegios católicos no son meros receptores pasivos de la educación sexual, y su agencia se expresa en su modo de tomar distancia de la educación sexual que reciben, aceptando y cuestionando algunas de las normas que aquella moviliza o que ven representadas en otros sujetos. Ahora bien, este posicionamiento se sostiene sobre una cartografía de diferencias, que permiten distinguir la propia postura de la de otros. El Ego se entiende diferenciado del Alter, sin embargo, siguen estando ocultas ciertas formas que puede adoptar la alteridad.

Tal como lo propone Schütz (1972), son determinados eventos, disruptivos e inesperados, los que pueden hacer evidente el mundo de relaciones dadas por obvias para un determinado actor. Se podría pensar en una primera instancia que para la educación sexual de los colegios católicos la disrupción está ligada a los valores que propone la cultura, no obstante, en este caso la religión no es lo más relevante para el posicionamiento de los estudiantes, sino el modo como los sujetos encarnan los valores, algo que está profundamente ligado a una frontera social todavía irreflexiva.

El punto de vista crítico que movilizan los jóvenes no alcanza a mirar las condiciones materiales y de capital cultural que influyen en cómo se experimenta la individualidad y la sexualidad. Volviendo sobre los ejemplos mostrados en el artículo no todos los sujetos se comparan con todos los sujetos, no todos comparten las mismas experiencias formativas o no todos comparten las mismas condiciones materiales para practicar la sexualidad. Si esta última siempre requiere del encuentro de un Alter y un Ego, es fácil comprender por qué la ausencia de determinadas experiencias, de determinadas miradas, impiden desarrollar una idea de inclusión más completa que haga reflexiva la frontera donde se construye -contingentemente- la normalidad.

No se puede entender el proyecto del sujeto (Ego) sino es a partir de la cartografía de los Otros significativos (Alter) que le rodean. La diversidad actual, que depende de un modo de representar las diferencias morales, requiere también mirar lo que ocurre más allá de 
una frontera social. Probablemente comprender ese límite permitiría avanzar en preguntas morales y prácticas inclusivas más audaces en el futuro.

\section{Referencias}

Amsellem-Mainguy, Y. y Vuattoux, A. (2019). Sexualité juvénile et rapports de pouvoir: Réflexions sur les conditions d'une éducation à la sexualité. Mouvements, 99, 85-95. https://doi.org/10.3917/mouv.099.0085

Astudillo, P. (2016). La inestable aceptación de la homosexualidad. El caso de las escuelas católicas de élite en Santiago de Chile. Revista Latinoamericana de Educación Inclusiva, 10(2), 21-37. https://doi.org/10.4067/S07 18-73782016000200003

Barrientos, J., Echague, C., Matus, C. y Astudillo, P. (2019). Homophobic violence against LGBTQ+ youth in a Chilean School. En J. I. Kjaran y H. Stauston (Eds.), School as queer transformative spaces (pp. 143-157). Londres: Routledge.

https://doi.org/10.4324/9781351028820-9

Bericat, E. (2000). La sociología de la emoción y la emoción en la sociología. Papers, 62, 145-176. https://doi.org/10.5565/rev/papers/v62no.1070

Bourdieu, P. (2012). La distinción. Criterios y bases sociales del gusto. Madrid: Taurus.

Bozon, M. (2009). Sociologie de la sexualité. París: Armand Colin.

Butler, J. (2018). El género en disputa. El feminismo y la subversión de la identidad. Barcelona: Paidós.

Canales, M. (2013). Análisis sociológico del habla. En M. Canales (Coord.), Escucha de la escucha. Análisis e interpretación en la investigación cualitativa (pp. 171-188). Santiago de Chile: Lom.

Collins, P. y Bilge, S. (2016). Intersectionality. Cambridge: Polity Press.

Connell, R. y Pearse, R. (2018). Género. Desde una perspectiva global. Valencia: PUV.

Cover, R. (2019). Emergent identities. New sexualities, genders and relationships in a digital era. Nueva York, NY: Routledge. https://doi.org/10.4324/9781315104348

Dubet, F. (2010). La sociología de la experiencia. Madrid: Editorial Complutense.

Dubet, F. (2013). Pour-quoi à moi ? L'expérience des discriminations. París: Payot.

Dubet, F. y Martuccelli, D. (1996). A l'école. Sociologie de l'expérience scolaire. París: Seuil.

Figueroa, E. (2012). Política pública de educación sexual en Chile: actores y tensión entre el derecho a la información vs. la libertad de elección. Estado, Gobierno, Gestión Pública, 19, 105-131. https://doi.org/10.5354/0717-6759.2012.25861

Giddens, A. (2006). La transformación de la intimidad. Sexualidad, amor y erotismo en las sociedades modernas. Madrid: Cátedra.

Hubbard, P. (2002). Sexing the self: Geographies of engagement and encounter. Social and Cultural Geography, 3(4) 365-381. https://doi.org/10.1080/1464936021000032478

Illouz, E. (2015). Why love hurts. A sociological explanation. Cambridge: Polity Press.

Le Mat, A. (2016). Education sexuelle. En J. Rennes (Dir.), Encyclopédie critique du genre (pp. 232241). París: La Découverte.

Madrid, S. (2016). La formación de masculinidades hegemónicas en la clase dominante: El caso de la sexualidad en los colegios privados de élite en Chile. Sexualidad, Salud y Sociedad, 22, 369398. https://doi.org/10.1590/1984-6487.sess.2016.22.17.a 
Márquez, Y., Gutiérrez-Barroso, J. y Gómez-Galdona, N. (2017). Equidad, género y diversidad en educación. European, Scientific Journal, 13(7), 300-319.

https://doi.org/10.19044/esj.2017.v13n7p300

Maturana, C., Kaeufer, A., Riquelme, C., Silva, M. P., Osorio, M. y Torres, N. (2016). Conocimientos sobre identidad sexual de profesores y profesoras: ¿Barreras o facilitadores de construcción identitaria?. Revista Latinoamericana de Educación Inclusiva, 10(2), 53-71. https://doi.org/10.4067/S0718-73782016000200005

Miller, S. J (2016). Teaching, affirming and recognizing trans and gender creative youth. Nueva York, NY: Palgrave Macmillan. https://doi.org/10.1057/978-1-137-56766-6

Montero-Ossandón, L. E., Valverde-Forttes, P., Dois-Castellón, A., Bicocca-Gino, M. y Domínguez-Hidalgo, A. (2017). La educación sexual: Un desafío para la educación católica. Educación y Educadores, 20(3), 343-363. https://doi.org/10.5294/edu.2017.20.3.1

Morgade, G. (2016). Educación sexual integral con perspectiva de género: La lupa de la ESI en el aula. Rosario: Homo Sapiens.

Palma, I., Reyes, D. y Moreno, C. (2013). Educación sexual en Chile: Pluralismo y libertad de elección que esconde una propuesta gubernamental conservadora. Política Educativa, 49, 1424.

Rojas, M. T., Fernández, M. B., Astudillo, P., Stefoni, C., Salinas, P. y Valdebenito, M. J. (2019). La inclusión de estudiantes LGBTI en las escuelas chilenas: Entre invisibilización y reconocimiento social. Pensamiento Educativo. Revista de Investigación Educacional Latinoamericana, 56(1), 1-14. https://doi.org/10.7764/PEL.56.1.2019.3

Rubin, G. (1984). Thinking sex: Notes for a radical theory of the politics of sexuality. En C. Vance (Ed.), Pleasure and danger. Exploring female sexuality (pp. 143-178). Londres: Routledge.

Scott, J. (1991). The evidence of experience. Critical Inquiry, 17(4), 773-791. https://doi.org/10.1086/448612

Schütz, (1972). Fenomenología del mundo social. Buenos Aires: Paidós.

Singly F. (2010). Les uns avec les autres. Quand l'individualisme crée du lien. París: Pluriel.

Staunston, H. (2019). Challenging gender and sexuality-based discrimination in UK schools. Young women's experiences of illegitimation and resistance. En J. I. Kjaran y H. Stauston, (Eds.), School as queer transformative spaces (pp. 123-142). Londres: Routledge. https://doi.org/10.4324/9781351028820-8

Thumala, M. A. (2007). Riqueza y piedad. El catolicismo de la élite económica chilena. Santiago de Chile: Debate.

\section{Breve CV del autor}

\section{Pablo Astudillo}

Sociólogo, Máster en sociología, especialidad Género, Política y Sexualidad de la Escuela de Altos Estudios en Ciencias Sociales (EHESS) de París. Doctor en sociología de la Universidad Paris Descartes (Paris V). Académico del Departamento de Política Educativa y Desarrollo Escolar de la Facultad de Educación de la Universidad Alberto Hurtado. Investigador especializado en temáticas de género, sexualidad, diferenciación social y educación. Ha publicado artículos en relación a la diferenciación social en los espacios de sociabilidad homosexual y actualmente se ha especializado en cuestiones relativas a la educación sexual y la inclusión de la diversidad sexual y de género en escuelas 
Revista Latinoamericana de Educación Inclusiva

chilenas. ORCID ID: https://orcid.org/0000-0002-7424-4038. Email: pastudil@uahurtado.cl 